\title{
Dynamic force analysis of a six-link planar mechanism
}

\author{
Yertugan Umbetkulov ${ }^{1}$, Yerlan Yeleukulov ${ }^{1}$, Alfiya Atalykova ${ }^{1}$, Gulbarshyn Smailova ${ }^{2}$, \\ Gulmira Iskakova ${ }^{1}$, Algazy Zhauyt ${ }^{1, *}$ and Gulama-Garip Alisher Ibrayev ${ }^{3}$ \\ ${ }^{1}$ Almaty University of Power Engineering and Telecommunication, Almaty 050013, Kazakhstan \\ ${ }^{2}$ Kazakh National Agrarian University, Almaty 050010, Kazakhstan \\ ${ }^{3}$ Al-Farabi Kazakh National University, Almaty 050040, Kazakhstan
}

\begin{abstract}
In this paper is studied a vibratory conveyor that is placed on an elastic base. Using the closed contours method it was determined the system that needs to be solved to obtain graphical representation for the generalized coordinates determining the position of the mechanical system elements. The shaking conveyor represents the chase hanged or supported to the fixed section. The chase commits oscillating motions hereupon the cargo which is in the chase, migrates concerning to the chase. The nature of the flow and its parameters are determined by the nature of the oscillating committed by the chase. Installation causes fluctuations fixed tray. Uniformly distributed load on the tray acts in each element of the mechanism. A proper dynamic model has been developed within MSC ADAMS and Maple 18. Simulation tests have been carried out and results are discussed to validate the proposed design solution.
\end{abstract}

\section{Introduction}

The big application in various fields of the industry was received by the shaking conveyors applied to transportation of hot, poisonous, chemical aggressive cargoes by the supplement of complete tightness of their relocation [1], and also for transportation of the metallic cuttings damped with emulsion and oil, hot earth which has been beaten out from casting forms, small casting, foundry fusion mixture, etc. The shaking conveyor represents the chase hanged or supported to the fixed section. The chase commits oscillating motions hereupon the cargo which is in the chase, migrates concerning to the chase [2]. The nature of the flow and its parameters are determined by the nature of the oscillating committed by the chase. Shaking conveyors on the conditions of the chase flow and nature of cargo movement are subdivided on inertial (with variable and constant stress of cargo to the chase) in which [3] cargo under the influence of inertia force glides on the chase, and on vibrating in which cargo tears off the chase and migrates along the chase. The vibrating conveyors [4] are widely applied owing to a number of advantages in these latter days. The questions of the kinematic and dynamic study of the vibrating feeder intended for dosing of the fusion mixture loading of the melting furnaces of foundry production are considered in the presented work [5]. The principle of operation of the vibrating conveyor is described, and it

\footnotetext{
${ }^{*}$ Corresponding author: ali84jauit@mail.ru
} 
is devoted the kinematic analysis of the action. The differential equation of the link move of the reduction of the vibrating conveyor is considered in the difference method (the approximate method) a solution of the equation of move of the vibrating conveyor is resulted [6]. It is devoted to the analysis of the equations solutions of conveyor move. Here tables of the results and relocation drawing and velocity of the leading link depending on time are resulted [7].

\section{Kinematic analysis of six-bar linkage}

The equations for kinematic analysis are written and solved considering a Cartesian coordinate system, with origin the joint $\mathrm{O}$. The mechanism has two independent closed contours OABCEFO and OABCDGO. The vector equation corresponding to each above independent closed loop is (see Figure 1):

Table 1. The parameters of six-bar linkage.

\begin{tabular}{|l|l|l|c|c|c|c|c|c|}
\hline Dimensions & $l_{O A}$ & $l_{A B}$ & $l_{B C}=l_{K}$ & $l_{C D}$ & $l_{C E}$ & $l_{D G}$ & $l_{E F}$ & $a=O M$ \\
\hline $\mathrm{mm}$ & 60 & 430 & 75 & 1140 & 560 & 440 & 440 & 50 \\
\hline
\end{tabular}

For closed contour OABCEFO: $l_{1}+l_{2}+l_{k}+l_{3}^{\prime}=l_{7}+l_{4}$

and for closed contour OABCDGO: $l_{1}+l_{2}+l_{k}=l_{6}+l_{5}+l_{3}^{\prime \prime}$

The vector equations (1) and (2) are projected on the Cartesian reference system. It is obtained:

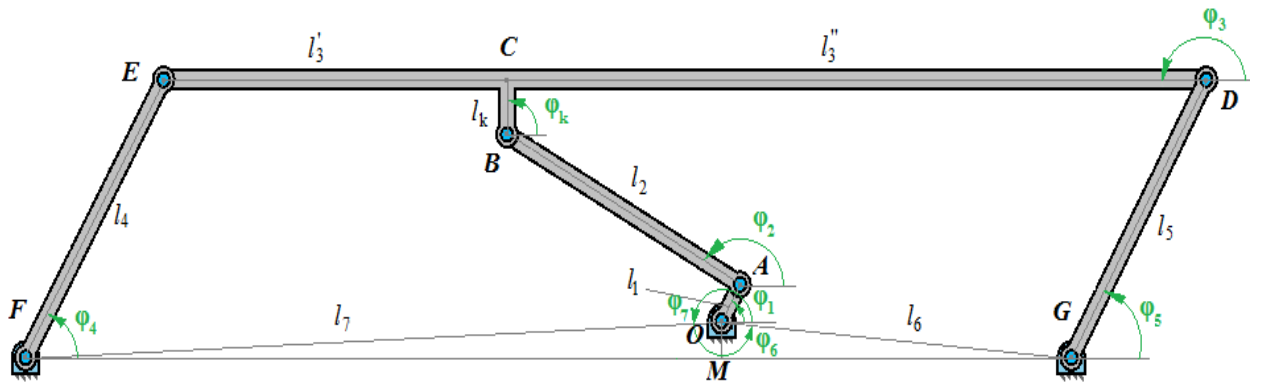

Fig. 1. Kinematic model of six-bar mechanism.

$$
\begin{aligned}
& l_{1} \cos \varphi_{1}+l_{2} \cos \varphi_{2}+l_{3}^{\prime} \cos \varphi_{3}=l_{7} \cos \varphi_{7}+l_{4} \cos \varphi_{4} \\
& l_{1} \sin \varphi_{1}+l_{2} \sin \varphi_{2}+l_{k}+l_{3}^{\prime} \sin \varphi_{3}=l_{7} \sin \varphi_{7}+l_{4} \sin \varphi_{4} \\
& l_{1} \cos \varphi_{1}+l_{2} \cos \varphi_{2}=l_{6} \cos \varphi_{6}+l_{5} \cos \varphi_{5}+l_{3}^{\prime \prime} \cos \varphi_{3} \\
& l_{1} \sin \varphi_{1}+l_{2} \sin \varphi_{2}+l_{k}=l_{6} \sin \varphi_{6}+l_{5} \sin \varphi_{5}+l_{3}^{\prime \prime} \sin \varphi_{3}
\end{aligned}
$$

ED since the joints are constantly moving horizontally $\varphi_{3}=180^{\circ}$ the angle remains unchanged. By agreement $l_{4}=l_{5}$, So $\varphi_{4}=\varphi_{5}, \varphi_{k}=90^{\circ}$, So, let's make it a little easier:

$$
l_{2} \cos \varphi_{2}-l_{4} \cos \varphi_{4}=l_{7} \cos \varphi_{7}-l_{1} \cos \varphi_{1}+l_{3}^{\prime}
$$




$$
\begin{aligned}
& l_{2} \sin \varphi_{2}-l_{4} \sin \varphi_{4}=l_{7} \sin \varphi_{7}-l_{1} \sin \varphi_{1}-l_{k} \\
& l_{2} \cos \varphi_{2}-l_{4} \cos \varphi_{4}=l_{6} \cos \varphi_{6}-l_{1} \cos \varphi_{1}-l_{3}^{\prime \prime} \\
& l_{2} \sin \varphi_{2}-l_{4} \sin \varphi_{4}=l_{6} \sin \varphi_{6}-l_{1} \sin \varphi_{1}-l_{k}
\end{aligned}
$$

Eq. (7-8) and Eq. (9-10) If we compare equations, this equations left sides equal to each other. Let's prove the equilibrium:

$$
\begin{aligned}
& l_{7} \cos \varphi_{7}-l_{1} \cos \varphi_{1}+l_{3}^{\prime}=l_{6} \cos \varphi_{6}-l_{1} \cos \varphi_{1}-l_{3}^{\prime \prime} \\
& l_{7} \sin \varphi_{7}-l_{1} \sin \varphi_{1}-l_{k}=l_{6} \sin \varphi_{6}-l_{1} \sin \varphi_{1}-l_{k}
\end{aligned}
$$

$l_{1} \sin \varphi_{1}, l_{k}$, and $l_{1} \cos \varphi_{1}$ after deduction, we get the following:

$$
\begin{aligned}
& l_{7} \cos \varphi_{7}+l_{3}^{\prime}=l_{6} \cos \varphi_{6}-l_{3}^{\prime \prime} \\
& l_{7} \sin \varphi_{7}=l_{6} \sin \varphi_{6}
\end{aligned}
$$

$\varphi_{6}=354^{\circ}$ and $\varphi_{7}=181^{\circ} 40^{\prime}, l_{7}=1325,6, \quad l_{6}=377,1, \quad l_{3}^{\prime}=560, \quad l_{3}^{\prime \prime}=1140$.

Solution of equations Eq. (13-14) $\varphi_{1}$ relative to the angle $\varphi_{2}$ and $\varphi_{4}$ determine the angles. For this Eq. (13-14) we introduce the notations $\mathrm{A}$ and $\mathrm{B}$ on the right-hand side of the system of equations. We know the values of $\mathrm{A}$ and $\mathrm{B}$ :

$$
\begin{aligned}
& A=l_{7} \cos \varphi_{7}-l_{1} \cos \varphi_{1}+l_{3}^{\prime} \\
& B=l_{7} \sin \varphi_{7}-l_{1} \sin \varphi_{1}-l_{k}
\end{aligned}
$$

Here are two equations with two unknowns:

$$
\begin{aligned}
& l_{2} \cos \varphi_{2}-l_{4} \cos \varphi_{4}=A \\
& l_{2} \sin \varphi_{2}-l_{4} \sin \varphi_{4}=B \\
& C=\frac{l_{2}^{2}}{2 A}-\frac{B^{2}}{2 A}+\frac{A}{2}-\frac{l_{4}^{2}}{2 A}
\end{aligned}
$$

Let's introduce some values:

$$
M=l_{4}^{2}\left(1+\frac{B^{2}}{A^{2}}\right), \quad N=2 B l_{4}\left(1-\frac{C}{A}\right), \quad P=B^{2}+C^{2}-l_{2}^{2}
$$

The resulting values represent Eq. (19) equation, we obtain the following:

$$
M \sin ^{2} \varphi_{4}+N \sin \varphi_{4}+P=0
$$

From $\varphi_{4}$ we define:

$$
\begin{gathered}
\sin \varphi_{4}=\frac{-N \pm \sqrt{N^{2}-4 M P}}{2 M}=W, W=W\left(\varphi_{1}\right) ; \varphi_{4}=\arcsin W+2 \pi n, \\
\varphi_{4}=f\left(\varphi_{1}\right)
\end{gathered}
$$


From this equation $\varphi_{2}$ we define:

$$
\begin{aligned}
\sin \varphi_{2}=\frac{\left(B+l_{4} \cdot W\right)}{l_{2}}, W=W\left(\varphi_{1}\right) ; \varphi_{2} & =\arcsin \left[\frac{\left(B+l_{4} \cdot W\right)}{l_{2}}+2 \pi n\right] \\
\varphi_{2} & =f\left(\varphi_{1}\right)
\end{aligned}
$$

The results of displacement analysis are used for velocity analysis. Differentiating equations (3) and (4) with respect to time and after rearranging terms it is obtained a set of two linear equations in the unknown angular velocities $\omega_{2}, \omega_{3}, \omega_{4}$ and $\omega_{5}$. By solving this system the unknown velocities are determined as:

$$
\begin{aligned}
& \frac{\omega_{2}}{\omega_{1}}=u_{21}, \omega_{2}=\frac{d \varphi_{2}}{d t}=\frac{d \varphi_{2}}{d \varphi_{1}} \cdot \frac{d \varphi_{1}}{d t}=u_{21} \cdot \omega_{1} \\
& \frac{\omega_{3}}{\omega_{1}}=u_{31}, \omega_{3}=\frac{d \varphi_{3}}{d t}=\frac{d \varphi_{3}}{d \varphi_{1}} \cdot \frac{d \varphi_{1}}{d t}=u_{31} \cdot \omega_{1} \\
& \frac{\omega_{4}}{\omega_{1}}=u_{41}, \omega_{4}=\frac{d \varphi_{4}}{d t}=\frac{d \varphi_{4}}{d \varphi_{1}} \cdot \frac{d \varphi_{1}}{d t}=u_{41} \cdot \omega_{1} \\
& \frac{\omega_{5}}{\omega_{1}}=u_{51}, \omega_{5}=\frac{d \varphi_{5}}{d t}=\frac{d \varphi_{5}}{d \varphi_{1}} \cdot \frac{d \varphi_{1}}{d t}=u_{51} \cdot \omega_{1}
\end{aligned}
$$

The results of kinematic analysis are furthermore used to calculate the accelerations of the link mass centers needed to perform the kinetostatic analysis of the mechanism.

\section{Dynamic force analysis of six-bar mechanism}

When the inertia forces are considered in the analysis of the mechanism, the analysis is known as dynamic force analysis. Now applying D'Alembert principle one may reduce a dynamic system into an equivalent static system and use the techniques used in static force analysis to study the system. In the six-bar mechanism shown in Figure 1, Link 1 is the ground link (sometimes called the frame or fixed link), and is assumed to be motionless. Links 4 and 5 each rotate relative to the ground link about fixed pivots (F and G). Link 3 is called the coupler link, and is the only link that can trace paths of arbitrary shape (because it is not rotating about a fixed pivot). Usually one of the "grounded links" (link 1) serves as the input link, which is the link which may either be turned by hand, or perhaps driven by an electric motor or a hydraulic or pneumatic cylinder. For this purpose, D'Alembert's Principle which states that the inertia forces and couples, and the external forces and torques on the body together give statically equilibrium, was considered. The inertia forces $P_{i j}$ and inertia moments $M_{i j}$ are given by. From the free body diagrams (see Figure 2) three static equilibrium equations, in terms of forces in the $\mathrm{X}$ and $\mathrm{Y}$ directions and moment about the center of gravity of the link, can be written for each link. For link 1, we get:

$$
\begin{aligned}
& P_{01, X}+P_{12, X}=m_{1} \cdot a_{G 1, X} \\
& P_{01, Y}+P_{12, Y}=m_{1} \cdot a_{G 1, Y}
\end{aligned}
$$




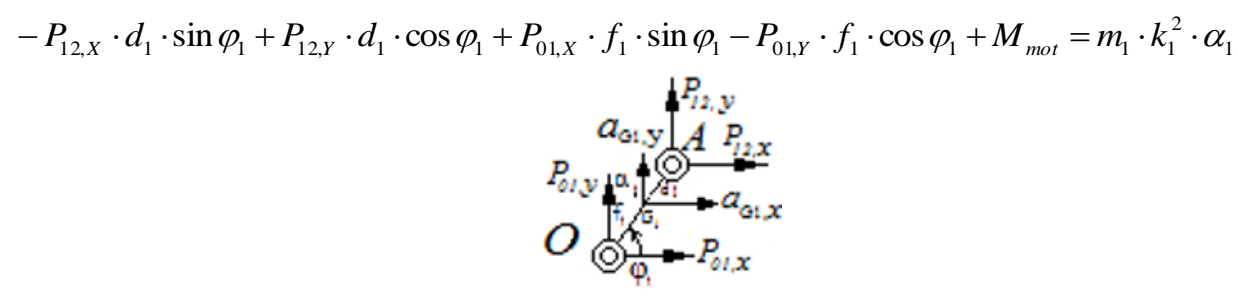

Fig. 2. The free-body diagrams of link 1 (input link).

At this particular instant the angles made are $\varphi_{1}$ the lengths of the links are known, the location of the respective center of mass is $G_{1}$ we also give the dimensions for this part that is, from $\mathrm{O}$ to $G_{1}$ as $d_{1}, G_{1}$ to A as $f_{1}$, and the input moment $M_{m o t}$. Let the acceleration components of the mass center be $a_{G 1, X}, a_{G 1, Y}$ and where $k_{1}$ is the radius and $\alpha_{1}$ is the angular acceleration (see Figure 2).

$$
\begin{gathered}
P_{23, X}-P_{21, X}=m_{2} \cdot a_{G 2, X} \\
P_{23, Y}-P_{21, Y}=m_{2} \cdot a_{G 2, Y} \\
-P_{23, X} \cdot d_{2} \cdot \sin \varphi_{2}-P_{23, Y} \cdot d_{2} \cdot \cos \varphi_{2}-P_{21, X} \cdot f_{2} \cdot \sin \varphi_{2}-P_{21, Y} \cdot f_{2} \cdot \cos \varphi_{2}+M_{2}=m_{2} \cdot k_{2}^{2} \cdot \alpha_{2}
\end{gathered}
$$

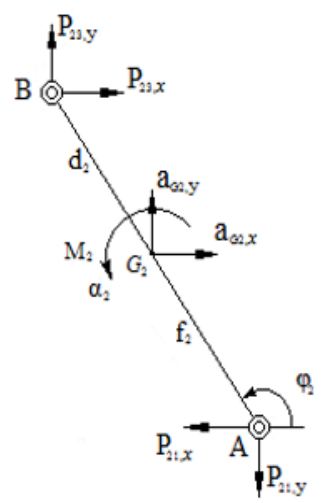

Fig. 3. The free-body diagrams of link 2 .

$$
\begin{gathered}
-P_{35, X}-P_{32, X}-P_{34, X}=m_{3} \cdot a_{G 3, X} \\
-P_{35, Y}-P_{34, Y}-P_{32, Y}=m_{3} \cdot a_{G 3, Y} \\
P_{35, Y} d_{3}-P_{32, X} B C+P_{32, Y} C G_{3}-P_{34, Y} f_{3}+M_{3}=m_{3} k_{3}^{2} \alpha_{3}
\end{gathered}
$$

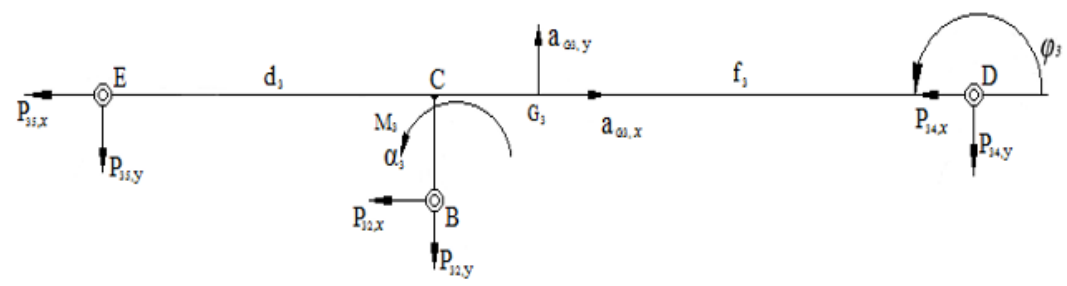

Fig. 4. The free-body diagrams of link 3. 
The angles made are equal $\varphi_{4}=\varphi_{5}$, the lengths of the links are known, We have already mentioned that since the kinematics is completely solved we all know the acceleration components and the angular accelerations. For the last link, exactly in the same procedure, we will get total equation in the $\mathrm{X}$ direction as plus $P_{43, X}$ and $P_{04, X}$ this must be equal to $m_{4} a_{G 4, X}$; in the Y direction, we will have plus $P_{43, Y}$ and $P_{04, Y}$ this is equal to $m_{4} a_{G 4, Y}$.

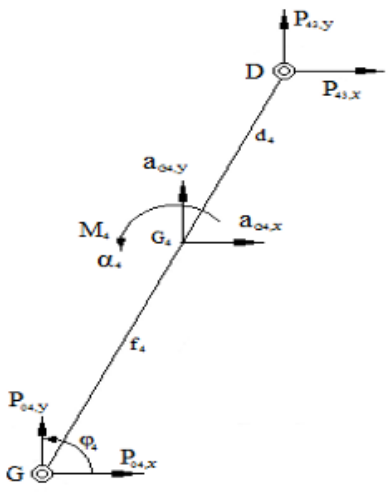

Fig. 5. The free-body diagrams of link 4 .

$$
\begin{gathered}
P_{43, X}+P_{04, X}=m_{4} \cdot a_{G 4, X} \\
P_{43, Y}+P_{04, Y}=m_{4} \cdot a_{G 4, Y} \\
-P_{43, X} \cdot d_{4} \cdot \sin \varphi_{4}+P_{43, Y} \cdot d_{4} \cdot \cos \varphi_{4}+P_{04, X} \cdot f_{4} \cdot \sin \varphi_{4}-P_{04, Y} \cdot f_{4} \cdot \cos \varphi_{4}+M_{4}=m_{4} \cdot k_{4}^{2} \cdot \alpha_{4} \\
P_{53, X}+P_{05, X}=m_{5} \cdot a_{G 5, X} \\
P_{53, Y}+P_{05, Y}=m_{5} \cdot a_{G 5, Y} \\
-P_{53, X} \cdot d_{5} \cdot \sin \varphi_{5}+P_{53, Y} \cdot d_{5} \cdot \cos \varphi_{5}+P_{05, X} \cdot f_{5} \cdot \sin \varphi_{5}-P_{05, Y} \cdot f_{5} \cdot \cos \varphi_{5}+M_{5}=m_{5} \cdot k_{5}^{2} \cdot \alpha_{5}
\end{gathered}
$$

Fig. 6. The free-body diagrams of link 5 .

There are fifteen unknowns present in Eqs. (28-42), and $P_{01, X}, P_{01, Y}, P_{12, X}, P_{12, Y}, P_{23, X}$, $P_{23, Y}, P_{35, X}, P_{35, Y}, P_{34, X}, P_{34, Y}, P_{04, X}, P_{04, Y}, P_{05, X}, P_{05, Y}, M_{m o t}$. These fifteen equations are represented in matrix form as (Eq. 43):

$$
D * g=b
$$

where 


$$
\left[\begin{array}{ccccccccccccccc}
1 & 0 & 1 & 0 & 0 & 0 & 0 & 0 & 0 & 0 & 0 & 0 & 0 & 0 & 0 \\
0 & 1 & 0 & 1 & 0 & 0 & 0 & 0 & 0 & 0 & 0 & 0 & 0 & 0 & 0 \\
-P_{01, X} & -P_{01, Y} & -P_{12, X} & P_{01, Y} & 0 & 0 & 0 & 0 & 0 & 0 & 0 & 0 & 0 & 0 & 1 \\
0 & 0 & -1 & 0 & 1 & 0 & 0 & 0 & 0 & 0 & 0 & 0 & 0 & 0 & 0 \\
0 & 0 & 0 & -1 & 0 & 1 & 0 & 0 & 0 & 0 & 0 & 0 & 0 & 0 & 0 \\
0 & 0 & -P_{21, X} & -P_{21, Y} & -P_{23, X} & -P_{21, Y} & 0 & 0 & 0 & 0 & 0 & 0 & 0 & 0 & 0 \\
0 & 0 & 0 & 0 & -1 & 0 & -1 & 0 & -1 & 0 & 0 & 0 & 0 & 0 & 0 \\
0 & 0 & 0 & 0 & 0 & -1 & 0 & -1 & 0 & -1 & 0 & 0 & 0 & 0 & 0 \\
0 & 0 & 0 & 0 & -P_{32, X} & P_{32, Y} & 0 & P_{35, Y} & 0 & -P_{34, Y} & 0 & 0 & 0 & 0 & 0 \\
0 & 0 & 0 & 0 & 0 & 0 & 0 & 0 & 1 & 0 & 1 & 0 & 0 & 0 & 0 \\
0 & 0 & 0 & 0 & 0 & 0 & 0 & 0 & 0 & 1 & 0 & 1 & 0 & 0 & 0 \\
0 & 0 & 0 & 0 & 0 & 0 & 0 & 0 & -P_{43, X} & P_{43, Y} & P_{04, X} & -P_{04, Y} & 0 & 0 & 0 \\
0 & 0 & 0 & 0 & 0 & 0 & 1 & 0 & 0 & 0 & 0 & 0 & 1 & 0 & 0 \\
0 & 0 & 0 & 0 & 0 & 0 & 0 & 1 & 0 & 0 & 0 & 0 & 0 & 1 & 0 \\
0 & 0 & 0 & 0 & 0 & 0 & -P_{53, X} & P_{53, Y} & 0 & 0 & 0 & 0 & P_{05, X} & -P_{05, Y} & 0
\end{array}\right] *\left[\begin{array}{c}
P_{01, X} \\
P_{01, Y} \\
P_{12, X} \\
P_{12, Y} \\
P_{23, X} \\
P_{23, Y} \\
P_{35, X} \\
P_{35, Y} \\
P_{34, X} \\
P_{34, Y} \\
P_{04, X} \\
P_{04, Y} \\
P_{05, X} \\
P_{05, Y} \\
M_{m o t}
\end{array}\right]\left[\begin{array}{l}
m_{1} \cdot a_{G 1, X} \\
m_{1} \cdot a_{G 1, Y} \\
m_{2} \cdot a_{G 2, X} \\
m_{2} \cdot a_{G 2, Y} \\
m_{2} \cdot a_{G 2, X} \\
m_{2} \cdot a_{G 2, Y} \\
m_{3} \cdot a_{G 3, X} \\
m_{3} \cdot a_{G 3, Y} \\
m_{3} \cdot a_{G 3, X} \\
m_{3} \cdot a_{G 3, Y} \\
m_{4} \cdot a_{G 4, X} \\
m_{4} \cdot a_{G 4, Y} \\
m_{5} \cdot a_{G 5, X} \\
m_{5} \cdot a_{G 5, Y} \\
m_{1} \cdot k_{1}^{2} \cdot a_{1}
\end{array}\right]
$$

The Eq. (43) then can be solved for the above mentioned unknowns.

This kinematic analysis is useful to find the velocity and acceleration of the mechanisms at the different position of the links. Moreover, this kinematic analysis is prerequisite for the dynamic analysis to find the joint forces and torque required for the various positions of manipulators.

\section{Numerical application}

The above equations have been implemented in a computer program using MSC ADAMS to generate the simulation presented throughout the paper. The main dimensions of the sixbar linkage (see Figure 7) used for kinematic analysis are inserted in Table 1. These dimensions have been chosen in order to assure an approximately constant and small velocity and, accordingly, a quick return suitable for deep drawing. The driver link 1 rotates with a constant angular velocity of $\omega_{1}=6.283185 \mathrm{rad} / \mathrm{s}$.

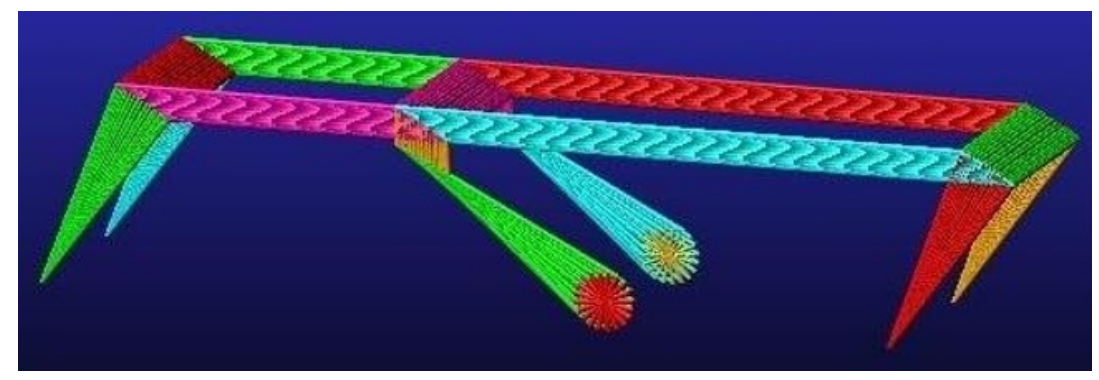

Fig. 7. Six-bar linkage motion simulation in MSC ADAMS.

The links positions for a complete rotation of the driver link 1 (the step of the angle is 151) are given in Figure 7. Some results for the dynamic simulation are shown in Figures 8 and 14. Figure 8-14 illustrates the $x$, y components of the joint reaction forces $P_{01, x y}, P_{12, x y}, P_{23, x y}$, $\mathrm{P}_{34, x y}, \mathrm{P}_{35, x y}, \mathrm{P}_{04, x y}$ and $\mathrm{P}_{05, x y}$ respectively versus crank angle with and without friction. In Figure 8 and Figure 14 is given the input torque versus crank angle with and without friction. In order to validate the simulation results obtained with the Maple 18 program, the 
values of the input torque without friction (Figures 8-14) are compared with the ones obtained from the conservation of energy law. It is remarked a very good agreement of the results.
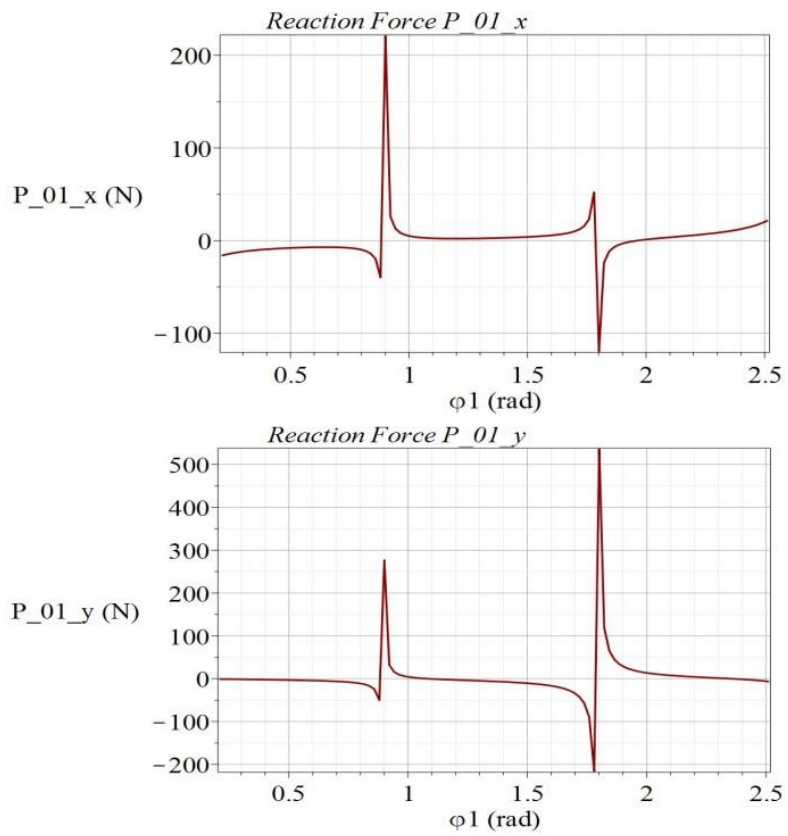

Fig. 8. Computed plot of the joint reaction forces $\mathrm{P}_{01} \mathrm{xy}$.
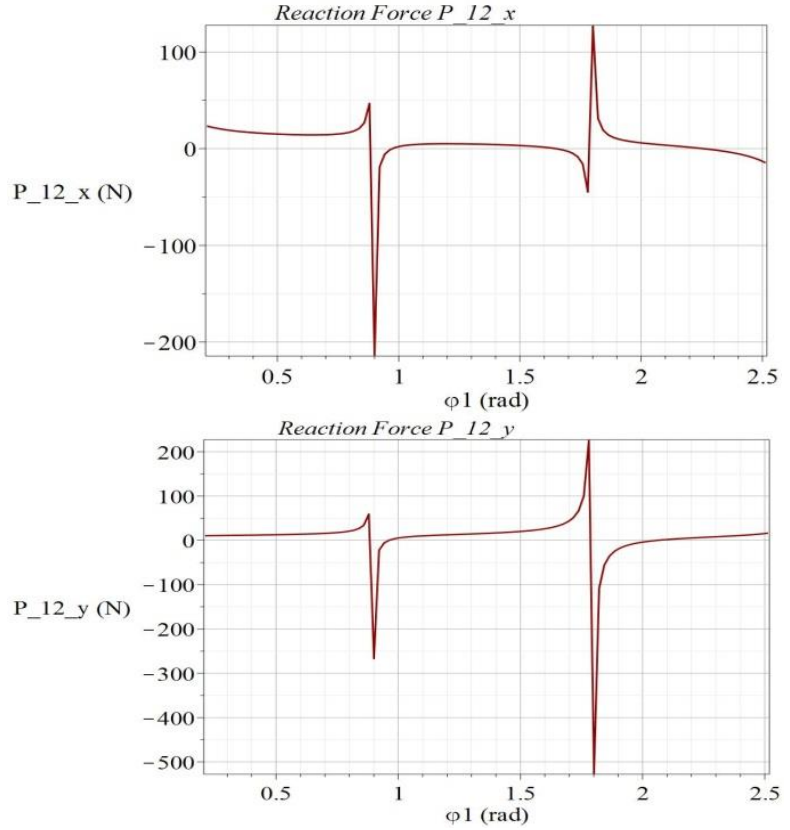

Fig. 9. Computed plot of the joint reaction forces $P_{12} x y$. 

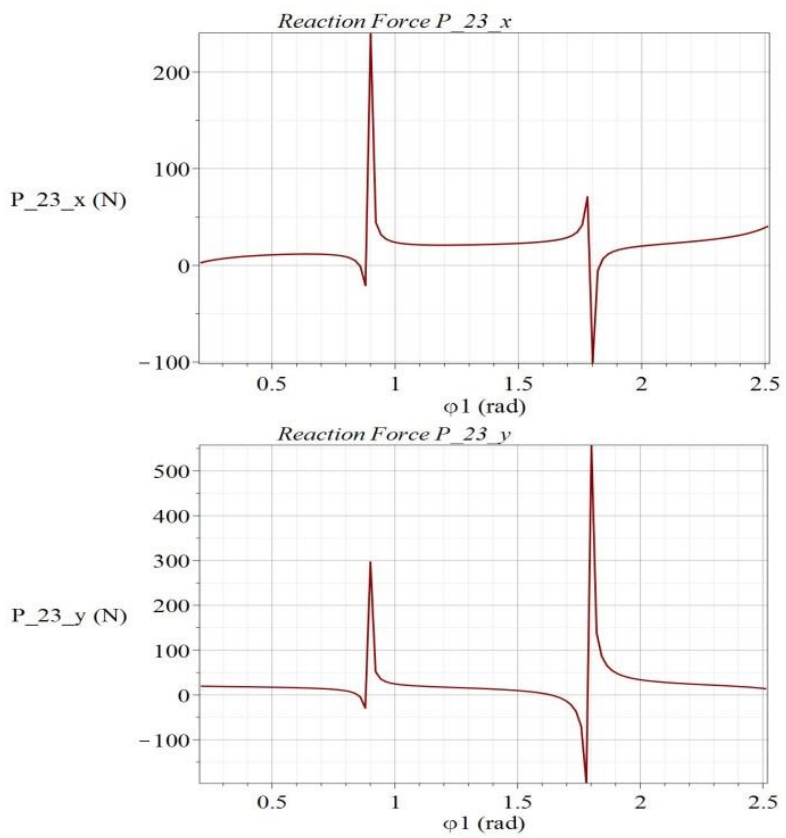

Fig. 10. Computed plot of the joint reaction forces $P_{23} x y$.
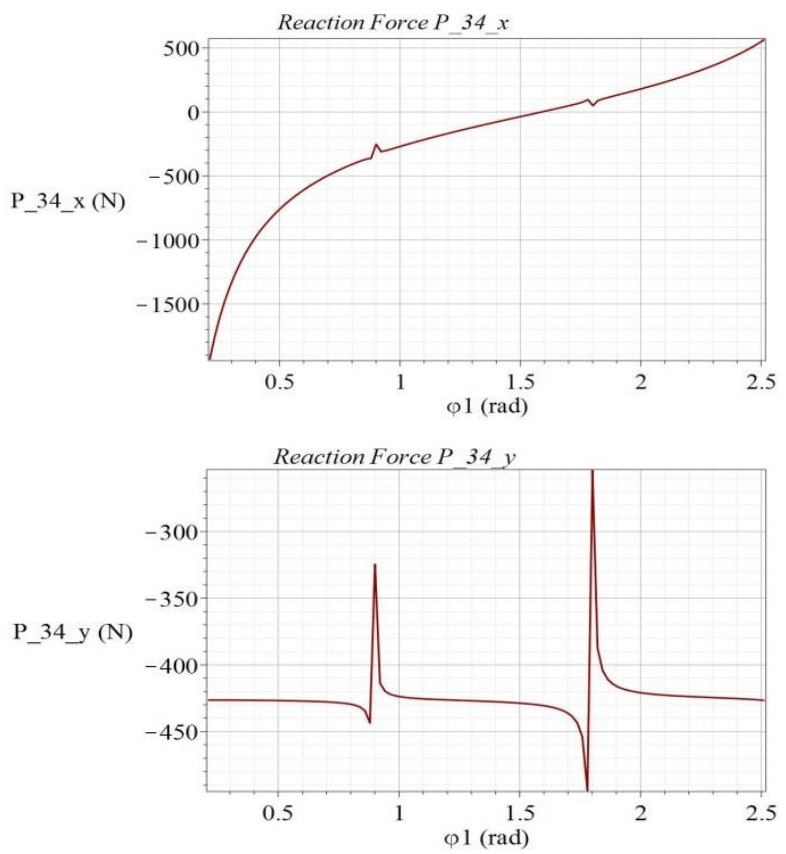

Fig. 11. Computed plot of the joint reaction forces $P_{34} x y$. 

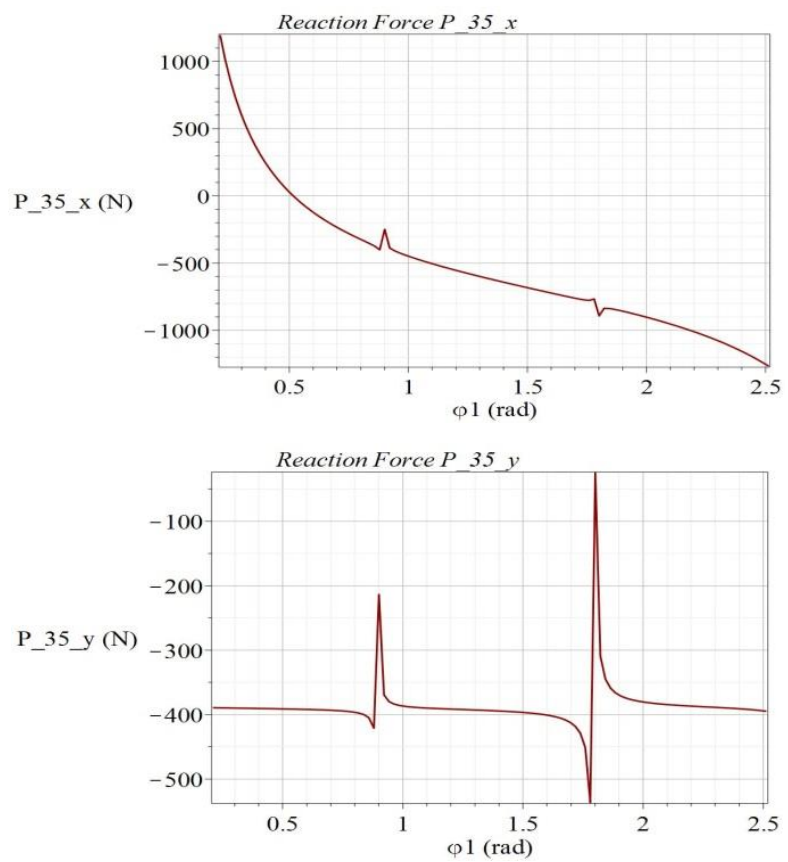

Fig. 12. Computed plot of the joint reaction forces $P_{35} x y$.
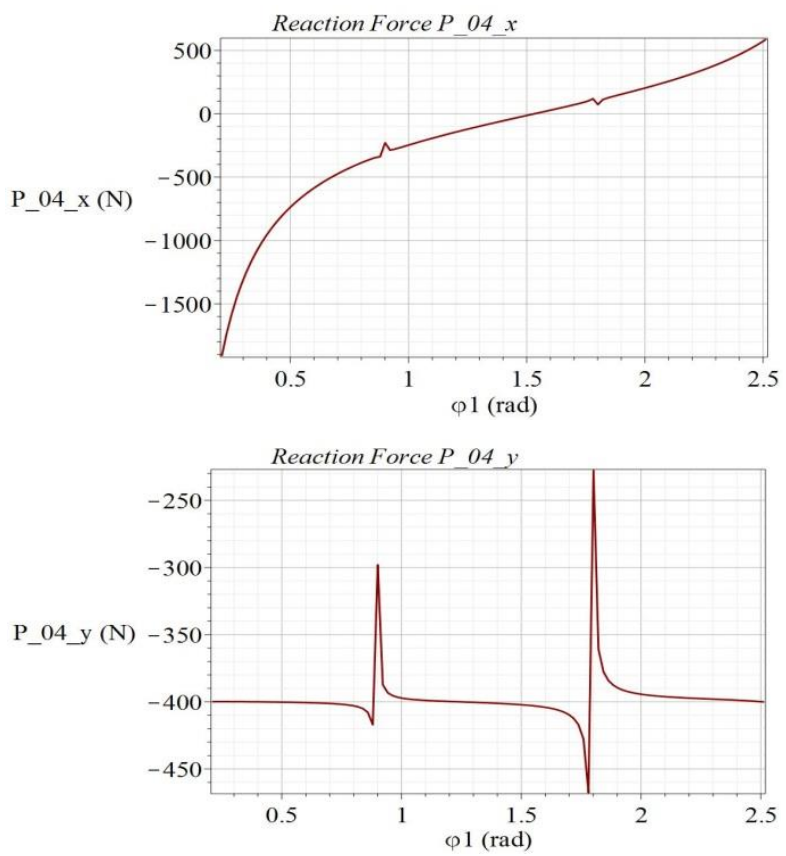

Fig. 13. Computed plot of the joint reaction forces $\mathrm{P}_{04} \mathrm{xy}$. 

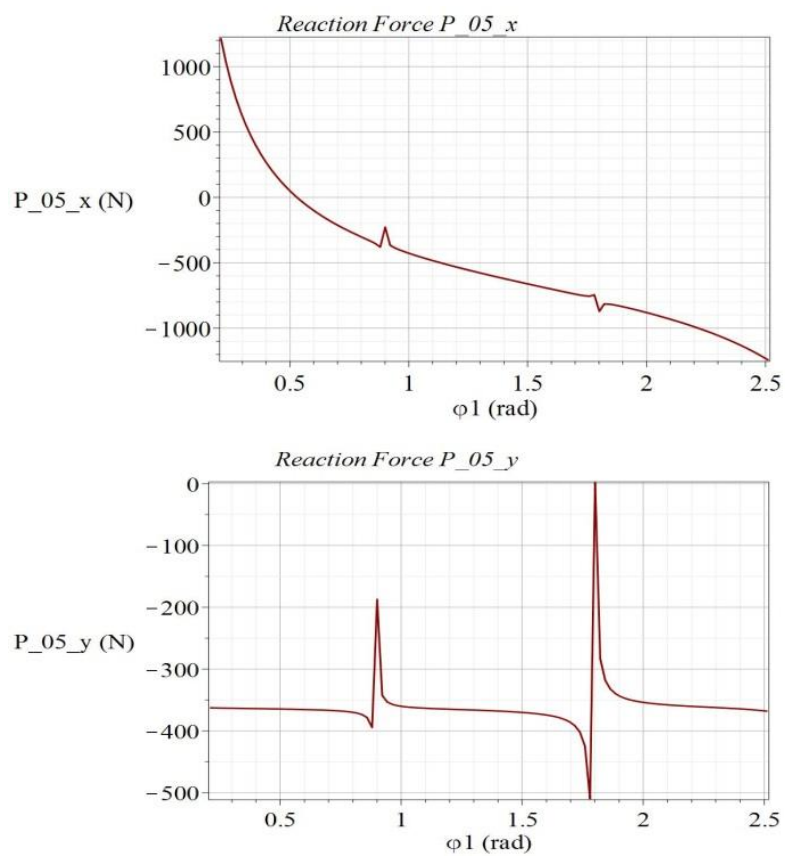

Fig. 14. Computed plot of the joint reaction forces $\mathrm{P}_{05} \mathrm{xy}$.

The analysis of obtained results are shown in the table:

Table 2. The analysis of obtained coordinates.

\begin{tabular}{|c|c|c|c|c|c|c|c|}
\hline $\begin{array}{c}\varphi_{1} \\
{[\mathrm{deg}]}\end{array}$ & $\varphi_{1}[\mathrm{rad}]$ & $\mathrm{X}_{\mathrm{A}}[\mathrm{m}]$ & $\mathrm{Y}_{\mathrm{A}}[\mathrm{m}]$ & $\mathrm{X}_{\mathrm{B}}[\mathrm{m}]$ & $\mathrm{Y}_{\mathrm{B}}[\mathrm{m}]$ & $\mathrm{X}_{\mathrm{C}}[\mathrm{m}]$ & $\mathrm{Y}_{\mathrm{C}}[\mathrm{m}]$ \\
\hline 135 & 2.355197551 & -0.042384089 & 0.04246868233 & -0.346742979 & -0.261283957 & -0.346742 & -0.186283 \\
\hline 225 & 3.924797551 & -0.042519357 & -0.0423332525 & 0.26086889526 & -0.347055312 & 0.2547769 & -0.272055 \\
\hline $\begin{array}{c}\varphi_{1} \\
{[\mathrm{deg}]}\end{array}$ & $\mathrm{X}_{\mathrm{D}}[\mathrm{m}]$ & $\mathrm{Y}_{\mathrm{D}}[\mathrm{m}]$ & $\mathrm{X}_{\mathrm{E}}[\mathrm{m}]$ & $\mathrm{Y}_{\mathrm{E}}[\mathrm{m}]$ & $\mathrm{X}_{\mathrm{F}}[\mathrm{m}]$ & $\mathrm{Y}_{\mathrm{G}}=\mathrm{Y}_{\mathrm{F}}[\mathrm{m}]$ & $\mathrm{X}_{\mathrm{G}}[\mathrm{m}]$ \\
\hline 135 & 0.0629833455 & 0.2614370037 & -0.906742979 & -0.1862839574 & -1.3246 & -0.05 & 0.3738 \\
\hline 225 & 0.0619913809 & -0.360443851 & -0.299131047 & -0.2720553120 & -1.3246 & -0.05 & 0.3738 \\
\hline
\end{tabular}

\section{Conclusions}

In the current study, the kinematic and kinetostatic analysis of a six-link planar mechanism for deep drawing has been investigated developing a Maple 18 program. An analytical method has been used for the determination of the displacement, velocity and acceleration of the links and the simulation of mechanism motion. The force analysis considering the joint friction is performed with an iterative procedure, applying the D'Alembert principle. It is observed that due to the low values of the friction forces and moments, the solution converges after one iteration step. The developed program can be useful for the optimization of the mechanism design considering different constraints.

\section{References}

1. Nguyen Phong Dien and Nguyen Van Khang, Dynamic force analysis of a six-link planar mechanism under consideration of friction at the joints, Vietnam Journal of Mechanics, 26, 2, pp. 65-75 (2004) 
2. S. Mitsi, I. Tsiafis, K. D. Bouzakis, Force analysis of six-bar linkage for mechanical presses considering joint frictions, Journal of the Balkan Tribological Association, 21, pp. 281-295 (2015)

3. Zhauyt Algazy, The substantiating of the dynamic parameters of the shaking conveyor mechanism, Vibroengineering Procedia, 5, pp. 15-20 (2015)

4. Lauw B. T. and Kinzel G. L., Computer-aided kinematic and force analyses for planar mechanisms, Proc., Computers in Engineering, ASME, pp 535-544 (1988)

5. A. Zhauyt, G. Mamatova, G. Abdugalieva, K. Alipov, A. Sakenova, A. Alimbetov, The kinematic analysis of flat leverage mechanism of the third class, IOP Conference Series: Materials Science and Engineering, 250 (2017), pp. 1-6 (2017)

6. B. Tultayev, G. Balbayev, A. Zhauyt, A kinematic analysis of flat leverage mechanism of the fourth class for manipulators, IOP Conference Series: Materials Science and Engineering, 230 (2017), pp. 1-7 (2017)

7. An Tzu Yang and ZhiShang Sun, Dynamic force and torque analysis of spherical four link mechanisms, journal of mechanisms, transmissions and automation in design, 105, 3, pp. 492-497 (1983) 